\title{
L'ACADÉMIE VÉTÉRINAIRE DE FRANCE ET SA CONTRIBUTION DANS LA PRÉSERVATION DE LA BIODIVERSITÉ ET DES ÉCOSYSTĖMES
}

\author{
THE FRENCH VETERINARY ACADEMY AND ITS CONTRIBUTION TO THE \\ PRESERVATION OF BIODIVERSITY AND ECOSYSTEMS
}

Par Bérangère $\mathrm{ABBA}^{1}$

(Discours d'ouverture de la Secrétaire d'État à La Biodiversité, Séance solennelle de l'Académie vétérinaire de France du 3 décembre 2020)

Mots-clés : Académie vétérinaire de France, Une seule santé, biodiversité, bien-être animal, vétérinaires

Key words : French veterinary Academy, One Health, biodiversity, animal welfare, veterinarians

\begin{abstract}
Monsieur le Président de l'Académie vétérinaire, Mesdames et Messieurs les académiciens, Mesdames et Messieurs.

Je suis très heureuse et honorée d'ouvrir cette séance de l'Académie vétérinaire de France. Ma présence parmi vous, illustre, je crois, le changement de regard que notre société porte aujourd'hui sur le règne animal. Je remercie donc pour cette invitation votre Président, Jean-Luc Angot, dont je connais et salue son implication personnelle dans les questions de biodiversité et de préservation des écosystèmes que la crise que nous vivons, sanitaire, économique et environnementale, l'a mise au coeur du débat public.
\end{abstract}

\section{BIODIVERSITÉ ET ÉMERGENCE DES PANDÉMIES}

Le lien entre perturbation de la biodiversité et émergence des pandémies est aujourd'hui largement documenté. Le dernier rapport de l'IPBES confirme que les activités humaines telles que le commerce des espèces sauvages, l'agriculture intensive, avec pour corollaire la déforestation et la dégradation des habitats naturels et l'urbanisation, en sont directement à l'origine. Il ne s'agit donc pas de chercher l'origine de nos problèmes dans l'écaille du pangolin ou sous l'aile de la chauve-souris, mais bien directement dans notre lien à la Nature. Cette situation à laquelle nous faisons face nous oblige à repenser le lien entre santés animale, humaine et environnementale. Vous êtes nombreux ici à travailler de longue date sur ce concept «One Health / Une seule santé » et à le porter dans les instances nationales et internationales. Cette intégration des enjeux de préservation des espèces et de leurs milieux dans toutes les politiques publiques, que ce soit en interministériel au niveau national jusque dans les plus hautes organisations internationales (l'OMS, l'OIE, la FAO et le PNUE), c'est tout le sens de ma mission. Comme de transposer ces concepts de manière concrète et opérationnelle. Je sais pouvoir compter sur vos réflexions, notamment au sein de la commission biodiversité que vous avez mise en place, pour être force de propositions.

\section{PLAN NATIONAL SANTÉ-ENVIRONNEMENT}

Le plan national santé-environnement, qui doit être adopté d'ici à la fin de l'année, a été enrichi de cette réflexion. Il prévoit une meilleure formation des professionnels de santé, la mise en place deux programmes prioritaires de recherche (l'un sur l'exposome et l'autre sur les pathogènes émergents) et une meilleure surveillance de la santé de la faune sauvage. Les agents de l'Office français de la biodiversité et son réseau SAGIR sont pleinement mobilisés et j'ai aujourd'hui besoin des vétérinaires pour une analyse croisée des enjeux de santé. L'année 2021 sera l'année de construction de notre stratégie nationale pour la biodiversité pour la prochaine décennie. Elle sera l'occasion d'introduire cette approche unifiée de la santé et de lui donner corps. Je compte sur vous pour y contribuer.

1. Ministère de la Transition Ecologique, 246 bd St Germain, 75007, Paris 


\section{BIEN-ÊTRE ANIMAL}

La question du bien-être animal et de la protection de la faune sauvage appelle également aussi vos éclairages. Je suis mobilisée aux côtés de Barbara Pompili (Ministre de la transition écologique, ndlr) sur l'itinérance de la faune sauvage captive dans les cirques. J'ai dans mon périmètre ministériel un autre sujet de société qui nécessite d'objectiver et de dépassionner, c'est celui de la chasse et de la régulation de la faune. Vous êtes sans doute, comme moi, très souvent interpellés sur certaines pratiques qui heurtent aujourd'hui nos sensibilités. Deux espèces notamment me semblent tout à fait rentrer dans le besoin d'une analyse croisée entre enjeux de santé publique et intervention sur les écosystèmes : le renard et le blaireau. Ces espèces sont vectrices de maladies (échinococcose pour le renard et tuberculose pour le blaireau) mais ces espèces ont bien évidemment leur place dans le fonctionnement des écosystèmes, y compris pour réguler d'autres vecteurs de maladies. Il me semblerait intéressant que vous puissiez, dans le cadre de vos travaux, réfléchir, avec l'ANSES et l'OFB à ces questions.

Je vous remercie et vous souhaite une belle après-midi de réflexions et d'échanges et une pleine réussite dans vos travaux. 